\title{
Qualidade de vida de participantes de um programa de prevenção de quedas no município de Maceió
}

\author{
Quality of life of participants in a fall \\ prevetion program in the city of Maceió
}

\section{Felipe Lima Rebêlo' \\ Nicole Fernanda dos Santos Lima ${ }^{2}$ (b) Janinny Kelly Oliveira Costa ${ }^{3}$ (C) Jackelyne Carla Moreira dos Santos ${ }^{4}$ (1)}

1Autor para correspondência. Universidade Estadual de Ciências da Saúde de Alagoas e Centro Universitário Cesmac (Maceió). Alagoas, Brasil. feliperebelo_fisio@yahoo.com.br

¿2Universidade Estadual de Ciências da Saúde de Alagoas (Maceió). Alagoas, Brasil. nicole_limafe@hotmail.com ${ }^{3,4}$ Centro Universitário Cesmac (Maceió). Alagoas, Brasil. janinny_kelly@hotmail.com, jackelynecarlam@gmail.com

RESUMO | INTRODUÇÃo: Fatores sociais, psicológicos, físicos e ambientais, associados ao comprometimento funcional decorrente do envelhecimento, podem interferir na qualidade de vida do indivíduo que envelhece. OBJETIVO: Avaliar a qualidade de vida de participantes de um programa de prevenção de quedas na cidade de Maceió. MÉTODOS: Trata-se de um estudo observacional, de corte transversal, realizado com os dados dos participantes de um Programa de prevenção de quedas da cidade de Maceió, onde foram incluídos todos os participantes com 60 anos ou mais, que realizaram a avaliação antes do início das intervenções e tiveram seus dados registrados nos prontuários, sendo excluídos aqueles que não completaram toda a avaliação ou apresentavam erros de registros e os que apresentaram alteração cognitiva segundo o Mine exame do estado mental. Foram analisados os dados referentes as características socioeconômicas e demográficas (idade, sexo, escolaridade, renda salarial e participação em grupos de terceira idade); dados referentes à saúde (número de doenças associadas, prática de atividade física, percepção de saúde e histórico de quedas) e à avaliação da qualidade de vida, mensurada pelo Whoqolbref. Para elucidar a influência das diversas variáveis sobre os domínios do Whoqol-bref realizaram-se análises de regressão linear multivariada. RESULTADOS: A amostra final foi de 66 idosos $(70,28 \pm 9,2)$, com predomínio do sexo feminino 54 (81,8\%). As médias dos quatro domínios foram semelhantes, com superioridade para o psicológico. Houve associação significativa entre o escore total do Whoqol e boa percepção de saúde $(p<0,01)$ e escolaridade $(p<0,06)$; entre o domínio ambiental e repercussão funcional pós queda $(p<0,01)$ e escolaridade $(p=0,04)$; entre o domínio físico e boa percepção de saúde $(p<0,01)$, número de doenças $(p=0,03)$ e prática de atividade física $(p=0,02)$; entre o domínio psicológico e boa percepção de saúde $(p<0,01)$ e escolaridade $(p=0,04)$. CONCLUSÃO: A qualidade de vida dos participantes apresentou equilíbrio entre os domínios físico, psicológico, social e ambiental, sendo superior no domínio psicológico. Associou-se a fatores como boa percepção de saúde, escolaridade, prática de atividade física, número de doenças associadas e presença de repercussão funcional pós queda.

PALAVRAS-CHAVE: Idoso. Qualidade de vida. Acidentes por Quedas.
ABSTRACT I INTRODUCTION: Social, psychological, physical, and environmental factors, associated with the functional impairment resulting from aging, can interfere with the quality of life of the aging individual. OBJECTIVE: To evaluate the quality of life of participants in a fall prevention program in the city of Maceió. METHODS: This is an observational, cross-sectional study carried out with data from participants in a fall prevention program in the city of Maceió, which included all participants aged 60 or over, who underwent the assessment before the start of interventions and had their data recorded in the medical records, excluding those who did not complete the entire evaluation or had errors in the records and those who presented cognitive impairment according to the Mine mental status exam. Data related to socioeconomic and demographic characteristics (age, sex, education, salary income, and participation in elderly groups) were analyzed; health data (number of associated diseases, physical activity, health perception, and history of falls) and quality of life assessment, measured by Whoqol-bref. To elucidate the influence of the various variables on the Whoqol-bref domains, multivariate linear regression analyzes were performed. RESULTS: The final sample was 66 elderly $(70.28 \pm 9.2)$, with a predominance of females $54(81.8 \%)$. The means of the four domains were similar, with superiority for the psychological. There was a significant association between the Whoqol total score and good perception of health $(p<0.01)$ and education $(p<0.06)$; between the environmental domain and functional repercussions after falling $(p<0.01)$ and education $(p=0.04)$; between the physical domain and good health perception $(p<0.01)$, number of diseases $(p=0.03$ ) and physical activity $(p=0.02)$; between the psychological domain and good perception of health $(p<0.01)$ and education $(p=$ 0.04). CONCLUSION: The quality of life of the participants showed a balance between the physical, psychological, social, and environmental domains, being superior in the psychological domain. It was associated with factors such as the good perception of health, education, physical activity, number of associated diseases, and the presence of functional repercussions after a fall. 


\section{Introdução}

A mudança no perfil demográfico populacional nos últimos anos é perceptível através da inversão da pirâmide etária, principalmente em países desenvolvidos e em desenvolvimento, nos quais evidencia-se o aumento da população idosa, decorrente de um declínio na taxa de natalidade e mortalidade, além dos avanços científicos no cuidado em saúde ${ }^{1}$.

Contudo, a longevidade não é sinônimo de uma veIhice com qualidade de vida; pois apesar do envelhecimento ser um processo dinâmico, progressivo e fisiológico, ele implica em alterações morfofuncionais no organismo que o torna mais passível às agressões intrínsecas e extrínsecas, o que pode levar a um prolongamento da vida acompanhado de comorbidades física, psíquica e social, podendo predispor os idosos a doenças crônicas e síndromes geriátricas²

A associação dessas doenças e síndromes intensificam a diminuição da capacidade funcional do idoso, e desencadeiam, principalmente, alterações de força e equilíbrio. Dentre esses processos, destaca-se a instabilidade postural, que deixa o idoso suscetível a quedas ${ }^{3}$.

Essas problemáticas afetam diretamente a saúde e a independência funcional da pessoa idosa, e consequentemente sua qualidade de vida, pois diminuem a habilidade do idoso em realizar suas atividades básicas e instrumentais de vida diária.

O conceito de qualidade de vida é amplo, e pode estar relacionado ao bem-estar pessoal, capacidade funcional, nível socioeconômico, estado emocional, apoio da família, a participação social, ao autocuidado, estado de saúde, intelectualidade, aspectos culturais, éticos e religiosos. Ou seja, envelhecer com qualidade de vida pressupõe um conceito multidimensional que é subjetivo, e varia de acordo com a individualidade de cada pessoa, dentro do seu contexto de vida, objetivos, expectativas e realizações ${ }^{5,6}$.

Assim, a manutenção de bons padrões de saúde, bem como a participação do idoso no meio social, permitindo-Ihe a percepção de sua inserção e importância na sociedade, predispõe o indivíduo a um processo de envelhecimento saudável, no seu sentido pleno.

Diante disso, ações voltadas para a promoção de um envelhecimento ativo, embasadas em estudos que as avaliem e as aprimorem, tornam-se cada vez mais relevantes. Através delas é possível incentivar a autonomia, a independência, a auto expressão e a reinserção social em busca de uma velhice bem-sucedida e com qualidade?

Dentro desse contexto, e entendendo que o evento de queda interfere negativamente na qualidade de vida do idoso, os programas de prevenção de quedas surgem para contribuir nesse aspecto, objetivando ainda a prevenção de novos episódios através de estímulo sobre a capacidade funcional.

Sendo assim, torna-se relevante a realização de estudos que avaliem a qualidade de vida em participantes de grupos de prevenção de quedas, para identificar possíveis alterações desse parâmetro, e, diante disso, sugerir propostas de ampliação da divulgação para aqueles que não se encontram inseridos neles.

Dessa forma, o presente estudo objetivou avaliar a qualidade de vida de participantes de um programa de prevenção do risco de quedas na cidade de Maceió, Alagoas, bem como analisar os fatores associados à qualidade de vida, para cada um dos domínios a ela relacionados (físico, psicológico, social e ambiental).

\section{Métodos}

Trata-se de um estudo observacional com abordagem quantitativa, descritiva e analítica, de corte transversal, realizado no município de Maceió, Alagoas, através dos dados dos idosos vinculados ao ambulatório de prevenção do risco de quedas da Santa Casa de Misericórdia de Maceió. A pesquisa foi realizada com os dados dos participantes que foram avaliados no período de 2014 a 2016. Este artigo é um braço de pesquisa do projeto guarda-chuva intitulado "Avaliação multidimensional da eficácia de um programa de prevenção de quedas em idosos: um projeto guarda-chuva". 
Foram incluídos todos os participantes com idade igual ou superior a 60 anos, que realizaram a avaliação antes do início das intervenções e tiveram seus dados registrados nos prontuários, sendo excluídos aqueles que não completaram toda a avaliação ou apresentavam erros de registros, e aqueles que apresentaram alteração cognitiva segundo o Mine exame do estado mental, considerando o ponto de corte de 18 para analfabetos, 24 para aqueles com até 4 anos de estudo e 26 para os que tinham mais de 4 anos de estudo.

O protocolo da pesquisa teve projeto aprovado pelo Comitê de Ética e Pesquisa do Centro Universitário Cesmac, sob o protocolo de número 1669-12.

A coleta de dados foi realizada no próprio ambulatório, diretamente nos prontuários dos idosos e as informações coletadas eram registradas em uma ficha de recolhimento de dados previamente elaborada. Para esta pesquisa, foram incluídos todos os idosos que participaram do projeto, compreendendo avaliações desde maio de 2011 a novembro de 2012.

Para participar do projeto, os indivíduos devem ter idade igual ou superior a 50 anos, sendo encaminhados por profissionais da área da saúde ou por inscrição própria. Para o estudo aqui apresentado, analisaram-se os dados apenas dos participantes com idade igual ou superior a 60 anos.

O projeto acontece uma vez por semana, no período vespertino, sendo as intervenções realizadas em grupos de 8 indivíduos, no máximo. A intervenção tem duração de duas horas, sendo dividida em dois momentos, com uma hora cada.

Os participantes, inicialmente, assistem palestras com temas direcionados a prevenção de quedas, ministradas por profissionais da equipe interdisciplinar de Geriatria e Gerontologia (médico geriatra, fisioterapeuta, psicólogo, nutricionista, assistente social, enfermeiro, educador físico, terapeuta ocupacional e fonoaudiólogo). Depois, são encaminhados a outra sala onde ocorre uma intervenção em grupo com o Fisioterapeuta responsável, que propõe exercícios direcionados para o treino de equilíbrio e estímulo cognitivo.
O projeto tem duração de quatro meses. Todos os participantes passam por uma avaliação multidimensional no início das atividades, onde são coletados todos os dados socioeconômicos e demográficos e avaliam-se: percepção de saúde, avaliação cognitiva, estado de humor, qualidade de vida, capacidade funcional, equilíbrio e mobilidade e medo de quedas. A mesma avaliação é repetida ao término das atividades.

Para este artigo, realizou-se uma abordagem transversal dos dados da avaliação inicial do projeto, e foram analisados os dados socioeconômicos e demográficos e as questões relacionadas à qualidade de vida, referentes ao questionário Word Health Organization Quality of Life - WHOQOL-BREF.

O WHOQOL-BREF é o questionário de qualidade de vida da Organização Mundial de Saúde, sendo um instrumento validado no Brasil, curto e autoaplicável. Consta de 26 questões divididas em quatro domínios: físico, psicológico, relações sociais e meio ambiente. Para cada aspecto da qualidade de vida contido no questionário, o indivíduo pode apresentar sua resposta por meio de escores que variam de 1 (um) a 5 (cinco), sendo a condição pior no escore 1 (um) e a melhor no escore 5 (cinco). Os resultados dos domínios apresentam valores entre 0 (zero) e 100 (cem), sendo piores os mais próximos de 0 (zero) e melhores, os mais próximos de 100 (cem) ${ }^{8}$.

A confecção do banco de dados foi realizada no Programa Excel $₫ 2003$ (ambiente Windows $\AA$ ), sendo a análise destes realizada no pacote estatístico SPSS $®$ (Statistical Package for Social Sciences) versão 15.0.

Para elucidar a influência das diversas variáveis socioeconômicas sobre os escores dos quatro domínios diferentes do WHOQOL e de sua média geral, realizaram-se análises de regressão linear multivariada. Para tal, primeiro os escores do domínio psicológico e físico foram transformados (reversão seguida por radiciação quadrada) para atenderem ao pressuposto da normalidade. Após isso, cinco análises de regressão linear multivariada, com modo de entrada passo a passo, foram conduzidas para identificar o modelo que melhor explicasse a variação nos escores dos 4 domínios e da média geral. A avaliação da correlação nos modelos foi feita pelo teste de Durbin-Watson e a capacidade preditiva dos modelos por meio dos valores de $\mathrm{R}^{2}$ ajustado. 


\section{Resultados}

O presente estudo constou de uma amostra final de 66 participantes (Figura 1) onde a média de idade foi de 70,28 anos $(\mathrm{dp}= \pm 9,2)$. Evidenciou-se predomínio do sexo feminino, $54(81,8 \%)$ e significativa proporção de alta escolaridade, com média de 8,8 anos de estudo ( $\mathrm{dp}= \pm 5,82)$, onde $30(45,5 \%)$ tinham mais de 9 anos de estudo, e 18 (27,3\%) tinham entre 4 e 9 anos de estudo, e, essa mesma proporção, 18 (27,3\%), tinham menos de 4 anos de estudo. Os dados referentes aos aspectos socioeconômicos mostram que a maioria são aposentados, correspondendo a 55 (88,3\%) do total de idosos. Assim como, 34 (51,5\%) apresentavam renda de um salário mínimo, e os demais, 32 (48,5\%), relataram renda maior que um salário mínimo, e 30 (45\%) afirmaram participar de atividades de socialização em algum grupo de idosos.

Figura 1. Fluxograma da coleta dos participantes de um programa de prevenção do risco de quedas na cidade de Maceió, Alagoas. 2014 a 2016

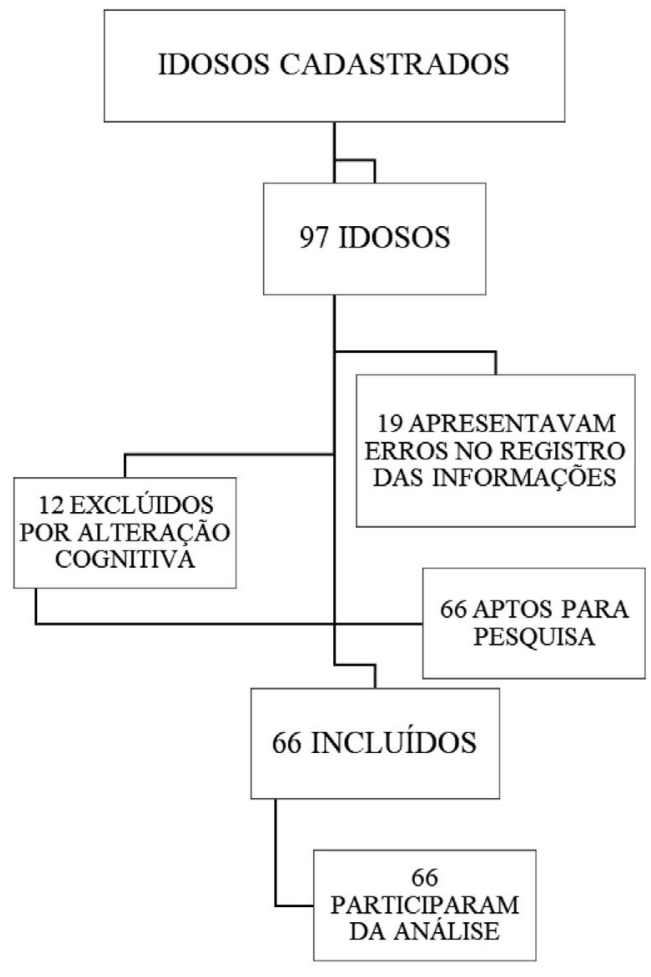

Em relação aos aspectos relacionados à saúde, a população estudada apresentou um número elevado de comorbidades associadas, onde a maioria, $41(62,1 \%)$ relatou ter mais de três doenças, sendo a média de 2,51 comorbidades ( $\mathrm{dp}= \pm 0,68)$. No que diz respeito a prática de atividade física, $40(60,6 \%)$ relataram não praticar nenhum tipo de atividade física, e, 53 (80,3\%) percebiam sua saúde como boa, na avaliação da percepção do estado de saúde. A maioria dos avaliados relatou ter caído no último ano, sendo a média de quedas de 1,48 $(\mathrm{dp}= \pm 1,8)$, variando de nenhuma até dez quedas, e, 55 (83,3\%) não apresentaram nenhum tipo de repercussão funcional após o evento de queda.

No que diz respeito aos resultados da avaliação da qualidade de vida, observou-se médias semelhantes para todos os domínios, sendo a maior média encontrada no domínio psíquico e a menor no físico (Tabela 1). 
Tabela 1. Distribuição das médias de Qualidade de Vida por domínios segundo o Whoqol-Bref. Maceió

\begin{tabular}{llllll}
\hline Domínio & Média & Mediana & Desvio Padrão & Valor mínimo & Valor máximo \\
& & & & & \\
\hline Físico & 60,25 & 66,07 & 20,05 & 7,14 & 92,86 \\
Psíquico & 65,72 & 66,66 & 17,53 & 16,67 & 95,83 \\
Social & 64,59 & 75 & 16,89 & 33,3 & 100 \\
Ambiental & 60,88 & 62,5 & 14,28 & 31,25 & 100 \\
Total & 65,36 & 67,02 & 12,99 & 30,69 & 93,79 \\
\hline
\end{tabular}

Fonte: Os autores (2021).

A análise de regressão linear multivariada mostrou que o modelo constituído pelo estado de saúde atual dos indivíduos e pelos anos de escolaridade foi o que melhor explicou a variação nos escores de WHOQOL total $\left(R^{2}\right.$ Ajust. = 0,268; Durbin-Watson = 2,01; $F=12,14 ; p<0,001$, (Tabela 2).

Tabela 2. Modelo multivariado que melhor explica a variação no WHOQOL Geral

\begin{tabular}{|c|c|c|c|c|c|}
\hline \multirow[b]{2}{*}{ Modelo } & \multicolumn{3}{|c|}{ Coeficientes } & \multicolumn{2}{|l|}{ IC95\% } \\
\hline & $\mathrm{B}$ & Erro Padrão & $\mathrm{P}$ & Limite Inferior & Limite Superior \\
\hline (Constante) & 36,63 & 6,5 & $<0,01$ & 23,61 & 49,65 \\
\hline Boa percepção (Saúde) & 12,37 & 3,5 & $<0,01$ & 5,34 & 19,4 \\
\hline Escolaridade & 0,714 & 0,25 & 0,06 & 0,21 & 1,21 \\
\hline
\end{tabular}

Fonte: Os autores (2021).

Na tabela 3, estão descritos os resultados da análise de regressão linear multivariada para o domínio ambiental, e mostrou que o modelo constituído pelos anos de escolaridade e a presença de repercussão funcional foi o que melhor explicou a variação nos escores desse domínio ( $R^{2}$ Ajust. $=0$; Durbin-Watson $=2,22 ; F=6,69 ; p=0,02$ ).

Tabela 3. Modelo multivariado que melhor explica a variação no domínio Ambiental (WHOQOL)

\begin{tabular}{|c|c|c|c|c|c|}
\hline \multirow[b]{2}{*}{ Modelo } & \multicolumn{3}{|c|}{ Coeficientes } & \multicolumn{2}{|l|}{ IC95\% } \\
\hline & $\mathrm{B}$ & Erro Padrão & $\mathrm{P}$ & Limite Inferior & Limite Superior \\
\hline (Constante) & 55,17 & 3,38 & $<0,01$ & 48,40 & 61,95 \\
\hline Escolaridade & 0,792 & 0,295 & $<0,01$ & 0,201 & 1,383 \\
\hline Repercussão Funcional & $-8,95$ & 4,41 & 0,04 & $-17,79$ & $-0,113$ \\
\hline
\end{tabular}

Fonte: Os autores (2021). 
Para a análise dos domínios físico e psicológico a intepretação de associação inversa ou direta devem ser interpretadas ao contrário (sinais de positividade devem ser interpretado como negatividade, e vice-versa), pois para as variáveis apresentarem uma distribuição normal, os dados desses domínios foram transformados (valores reversos).

A análise de regressão linear multivariada mostrou que o modelo constituído pelo estado de saúde atual dos indivíduos, pela pratica ou não de atividade física e pelo número de doenças, foi o que melhor explicou a variação nos escores do domínio Físico do WHOQOL ( $R^{2}$ Ajust. = 0,344; Durbin-Watson =1,96; $F=11,646 ; p<0,01$; Tabela 4).

Tabela 4. Modelo multivariado que melhor explica a variação no Domínio Físico (WHOQOL)

\begin{tabular}{|c|c|c|c|c|c|}
\hline \multirow[b]{2}{*}{ Modelo } & \multicolumn{2}{|c|}{ Coeficientes } & \multirow[b]{2}{*}{$P$} & \multicolumn{2}{|l|}{ IC95\% } \\
\hline & B & Erro Padrão & & Limite Inferior & Limite Superior \\
\hline (Constante) & 7,75 & 1,12 & $<0,01$ & 5,50 & 10,0 \\
\hline Boa percepção (saúde) & $-1,73$ & 0,47 & $<0,01$ & $-2,68$ & $-0,78$ \\
\hline Número de doenças & 0,85 & 0,39 & 0,03 & 0,05 & 1,64 \\
\hline Praticar atividade física & $-1,23$ & 0,393 & 0,02 & $-2,05$ & $-0,47$ \\
\hline
\end{tabular}

Fonte: Os autores (2021).

Na tabela 5, encontram-se os resultados da análise de regressão linear multivariada para o domínio psicológico, mostrou que o modelo constituído pelo estado de saúde atual dos indivíduos e pelos anos de escolaridade foi o que melhor explicou a variação nos escores desse domínio ( $R^{2}$ Ajust. $=0,207$; Durbin-Watson $=1,64 ; F=8,954 ; p$ $<0,001)$.

Tabela 5. Modelo multivariado que melhor explica a variação no Domínio Psicológico (WHOQOL)

\begin{tabular}{|c|c|c|c|c|c|}
\hline \multirow[b]{2}{*}{ Modelo } & \multicolumn{2}{|c|}{ Coeficientes } & \multirow[b]{2}{*}{$P$} & \multicolumn{2}{|l|}{ IC95\% } \\
\hline & B & Erro Padrão & & Limite Inferior & Limite Superior \\
\hline (Constante) & 8,69 & 0,85 & $<0,01$ & 6,98 & 10,39 \\
\hline Boa percepção (saúde) & $-1,52$ & 0,46 & $<0,01$ & $-2,44$ & $-0,60$ \\
\hline Escolaridade & $-0,06$ & 0,03 & 0,04 & $-0,13$ & $-0,002$ \\
\hline
\end{tabular}

Fonte: Os autores (2021).

A análise de regressão linear mostrou que nenhum modelo construído foi capaz de explicar adequadamente a variação nos escores do domínio social. 


\section{Discussão}

A análise dos dados do estudo aqui apresentado evidenciou um equilíbrio entre os domínios de qualidade de vida avaliados, sendo o psicológico discretamente superior aos demais. A qualidade de vida, associou-se a diversos fatores, e essas relações variaram a depender do domínio analisado.

Os achados desta pesquisa, assim como em outros estudos, revelaram situações semelhantes, no que diz respeito aos dados socioeconômicos e demográficos encontrados na sociedade brasileira, onde foi identificado predomínio da população feminina ${ }^{9}$ com média etária em torno dos 70 anos, vivendo com até um salário mínimo.

No que diz respeito à escolaridade, esse fator tem se mostrado como um importante determinante social para desfechos em saúde voltados para a qualidade de vida. No Brasil os idosos mais pobres têm piores condições de saúde e, apesar disso, usam menos esses serviços ${ }^{10}$.

Em relação à renda mensal, a população estudada, de modo geral, recebe até um salário mínimo. Ressaltase que, apesar de algumas pesquisas demonstrarem que a baixa renda reduz o bem-estar em idosos, no presente estudo, não foi encontrada associação significativa entre renda e qualidade de vida ${ }^{10}$.

Já a autopercepção de saúde mostrou-se associada a diversos domínios, sendo essa um desfecho pesquisado em diversos estudos de enfoque Gerontológico, e que tem se mostrado um método confiável para avaliação das condições de saúde na população idosa, sendo um parâmetro extremamente importante, uma vez que o indivíduo que envelhece, frequentemente remete a saúde como um fator importante para manutenção de qualidade de vida ${ }^{11}$.

Nessa pesquisa, identificou-se, para maioria dos avaliados, uma boa percepção de saúde, que também pode ser explicada pelo alto grau de escolaridade verificado na população estudada. Dados semelhantes foram encontrados no estudo de Joia et al. ${ }^{12}$ sobre condições associadas ao grau de satisfação com a vida em uma população de idosos.
O processo de envelhecimento humano é fisiológico, não sendo, portanto, caracterizado pela presença de doenças. No entanto, a diminuição da reserva funcional, característica dessa fase da vida predispõe o indivíduo ao surgimento de comorbidades associadas², fato evidenciado nesse estudo, onde a maioria dos sujeitos avaliados apresentou mais de três doenças.

Outro dado relevante, foi a alta proporção de sedentarismo, o que pode ser fator importante para risco de quedas, e interferir negativamente na qualidade de vida dos indivíduos. Ressalta-se que a atividade física regular exerce efeito positivo na preservação da massa óssea e a associação entre tratamento medicamentoso e atividade física é uma excelente forma de prevenir fraturas $\frac{13}{}$.

A regressão linear multivariada revelou associação significativa entre a média geral do Whoqol-bref com a escolaridade e a boa percepção da saúde. A avaliação da percepção de saúde bem como a da qualidade de vida são verificações subjetivas, de como o indivíduo que é avaliado percebe-se em relação a estes. Dessa forma, pode-se explicar a variação positiva, ou seja, maiores valores nas médias da qualidade de vida para os sujeitos que perceberam a saúde como boa. Associações positivas também foram encontradas entre boa percepção de saúde e os domínios físico e psicológico, e também podem ser interpretadas dessa forma.

Já em relação a escolaridade, foi verificado que a cada aumento em um ano de estudo, aumenta-se a média de qualidade de vida, tanto na média geral como nos domínios ambiental e psicológico. Resultados semelhantes foram encontrados por Pereira et al. ${ }^{14} \mathrm{e}$ Braga et al..$\frac{15}{1}$ onde os autores identificaram associação estatisticamente significante entre má percepção da qualidade de vida e menor nível de escolaridade.

Para a interpretação desse resultado, pode-se levar em conta que níveis de escolaridade mais baixo associam-se com piores indicadores de saúde e que baixo nível educacional também configuraria menores oportunidades de emprego, lazer e atividades sociais ${ }^{16}$, ou seja, interfere negativamente em todos os domínios avaliados pelo WHOQOL. 
Ter repercussão funcional pós queda também mostrou associação estatisticamente significativa com o domínio ambiental, onde a presença de repercussão funcional leva a uma redução nas médias da qualidade de vida neste domínio. Esta relação mostra a influência do ambiente do indivíduo sobre a qualidade de vida. De fato, um indivíduo que cursou com algum tipo de comprometimento funcional tende a se preocupar mais com o ambiente onde vive, destacando-se que condições ambientais precárias como moradias não adaptadas, infraestrutura deficiente e situações de violência influenciam a percepção da qualidade de vida ${ }^{17}$.

Ressalta-se ainda que um ambiente adequado para o idoso é aquele que, além de oferecer segurança para o mesmo, deve ser funcional, possibilitando ao indivíduo a execução de suas atividades de vida diária com maior independência e autonomia possível, proporcionando ainda a interação social ${ }^{18}$. O meio ambiente tem associação direta com a percepção acerca da qualidade de vida entre os indivíduos de maiores faixas etárias por está diretamente ligado com a prevenção de queda, interação social, capacidade funcional e bem-estar geral ${ }^{19}$.

Para o domínio físico, foi identificada associação positiva entre a boa percepção de saúde e a prática de atividade física, onde verifica-se tendência ao aumento das médias de qualidade de vida; e associação inversa com o número de doenças, onde a cada aumento unitário no número de doenças, verifica-se uma tendência a diminuição nas médias de qualidade de vida.

A prática regular de atividade física configura em mais vigor e energia para o desempenho das atividades do dia a dia. Conte e Lopes ${ }^{20}$, em estudo que avaliou a qualidade de vida em idosas, verificaram que as mulheres ativas obtiveram melhores médias no domínio físico, e atribuíram menor quadro álgico e mais disposição para as atividades diárias.

Já a associação inversa entre o maior número de doenças e o domínio físico, pode ser explicado pela maior tendência de comprometimento funcional, à medida que se aumenta o número de comorbidades²1.

A qualidade de vida sofre influência de diversos fatores, e no idoso, mais que em outros grupos etários, fatores físicos, psicológicos, sociais e culturais contribuem para mudanças na percepção desse desfecho.
Assim, avaliar e promover a saúde do idoso significa considerar variáveis de distintos campos do saber, numa atuação interdisciplinar e multidimensional22.

Destaca-se as limitações do estudo aqui apresentado, principalmente por se tratar de uma análise transversal de dados. Sugere-se, portanto, que estudos longitudinais e pesquisas que objetivem avaliar o efeito de estratégias direcionadas para otimização da qualidade de vida da pessoa idosa, como os programas de prevenção de quedas, sejam cada vez mais implementados, uma vez que esse deve ser o principal desfecho e objetivo final de qualquer intervenção em saúde.

\section{Conclusão}

Os resultados deste estudo revelaram médias semelhantes para os quatro domínios de qualidade de vida (físico, psicológico, ambiental e social). A boa percepção de saúde, a alta escolaridade e a prática de atividade física mostraram-se fatores que influenciam positivamente a qualidade de vida. Já a repercussão funcional pós-queda e um maior número de comorbidades, mostram influências negativas.

\section{Contribuições dos autores}

Rebêlo FL, Santos JCM e Costa JKO participaram da concepção, busca e análise estatística dos dados da pesquisa, interpretação dos resultados e redação do artigo científico. Lima NFS participou da redação e revisão do artigo científico.

\section{Conflitos de interesses}

Nenhum conflito financeiro, legal ou político envolvendo terceiros (governo, empresas e fundações privadas, etc.) foi declarado para nenhum aspecto do trabalho submetido (incluindo, mas não se limitando a subvenções e financiamentos, participação em conselho consultivo, desenho de estudo, preparação de manuscrito, análise estatística, etc.).

\section{Referências}

1. Miranda LCV, Soares SM, Silva PAB. Qualidade de vida e fatores associados em idosos de um Centro de Referência à Pessoa Idosa. Ciênc. saúde coletiva, 2016;21(11):3533-44. http://dx.doi. org/10.1590/1413-812320152111.21352015 
2. Macena WG, Hermano LO, Costa TC. Alterações fisiológicas decorrentes do envelhecimento. Mosaicum. 2018;15(27):223-38. https://doi.org/10.26893/rm.v15i27.64

3. Piovesam AC, Pivetta HMF, Peixoto JMB. Fatores que predispõem a quedas em idosos residentes na região oeste de Santa Maria, RS. Rev. bras. geriatr. gerontol. 2011;14(1):75-83. http://dx.doi.org/10.1590/S1809-98232011000100009

4. Freitas CV, Sarges ESNF, Moreira KECS, Carneiro SR. Avaliação de fragilidade, capacidade funcional e qualidade de vida dos idosos atendidos no ambulatório de geriatria de um hospital universitário. Rev. bras. geriatr. Gerontol. 2016;19(1):119-28. http://dx.doi.org/10.1590/1809-9823.2016.14244

5. Dawalibi NW, Anacleto GMC, Witter C, Goulart RMM, Aquino RC. Envelhecimento e qualidade de vida: análise da produção científica da SciELO. Estud. psicol. (Campinas). 2013;30(3):393-403. https://doi.org/10.1590/S0103-166X2013000300009

6. Dawalibi NW, Goulart RMM, Prearo LC. Fatores relacionados à qualidade de vida de idosos em programas para a Terceira idade. Ciênc. saúde coletiva. 2014;19(8):3505-12. https://doi. org/10.1590/1413-81232014198.21242013

7. Ferreira OGL, Maciel SC, Costa SMG, Silva AO, Moreira MASP. Envelhecimento ativo e sua relação com a independência funcional. Texto contexto - enferm. 2012;21(3):513-8. https://doi. org/10.1590/S0104-07072012000300004

8. The world Health Organization Quality of Life Assessment (WHOQOL): Position paper from the Health Organization. Soc. Sci. Med. 1995;41(10):1403-9. https://doi.org/10.1016/02779536(95)00112-k

9. Vieira LS, Gomes AP, Bierhals IO, Farías-Antúnez S, Ribeiro CG, Miranda V, et al. Quedas em idosos no Sul do Brasil: prevalência e determinantes. Rev. Saúde Pública. 2018;52(22). https://doi. org/10.11606/s1518-8787.2018052000103

10. Almeida A, Nunes BP, Duro SMS, Facchini LA. Determinantes socioeconômicos do acesso a serviços de saúde em idosos: revisão sistemática. Rev. Saúde Pública. 2017;51:50. https://doi. org/10.1590/s1518-8787.2017051006661

11. Krueg RR, Schneider IJC, Giehl MWC, Antes DL, Confortin SC, Mazo GZ, et al. Fatores sociodemográficos, comportamentais e de saúde associados à autopercepção de saúde positiva de idosos longevos residentes em Florianópolis, Santa Catarina. Rev. bras. epidemiol. 2018;21:e180004. http://dx.doi.org/10.1590/1980549720180004

12. Joia LC, Ruiz T, Donalisio MR. Condições associadas ao grau de satisfação com a vida entre a população de idosos. Rev. Saúde Pública. 2007; 41(1):131-8. https://doi.org/10.1590/S003489102007000100018
13. Gonçalves KS, Gaspar ID, Lemos J, Machado LMC, Siqueira PT, Lemos MLS. Comparação resultante de equilíbrio corporal e risco de queda entre idosos sedentário e ativos. Rev de trabaIhos acadêmicos - Universo Campos do Goytacazes [Internet]. 2017;1(8). Disponível em: http://revista.universo.edu.br/index. php?journal=1CAMPOSDOSGOYTACAZES2\&page $=$ article\&op $=-$ view\&path $\% 5 B \% 5 \mathrm{D}=6120$

14. Pereira RJ, Costa RMM, Francheschini SCC, Ribeiro RCL, Sampaio RF, Priore SE, et al. Contribuição dos domínios físico, social, psicológico e ambiental para a qualidade de vida global de idosos Rev. psiquiatr. Rio Gd. Sul. 2006;28(1):27-38. https://doi. org/10.1590/S0101-81082006000100005

15. Braga MCP, Casella MA, Campos MLN, Paiva SP. Qualidade de vida medida pelo Whoqol-bref: estudo com idosos residentes em Juiz de Fora/MG. Rev. de APS [Internet]. 2011;14(1):93-100. Disponível em: https://periodicos.ufjf.br/index.php/aps/article/ view/14649

16. Güths JFS, Jacob MHVM, Santos AMPV, Arossi GA, Béria JU. Perfil sociodemográfico, aspectos familiares, percepção de saúde, capacidade funcional e depressão em idosos institucionalizados no Litoral Norte do Rio Grande do Sul, Brasil. Rev. bras. geriatr. gerontol. 2017;20(2): 175-85. https://doi.org/10.1590/198122562017020.160058

17. Reis LA, Mascarenhas CHM, Torres GV. Avaliação da capacidade funcional em idosos institucionalizados no município de Jequié- Ba. Fiep Bulletin [Internet]. 2008;78(1):89-92. Disponível em: http://www.fiepbulletin.net/index.php/fiepbulletin/article/ view/3570/6966

18. Santos KA, Koszuoski R, Dias-da-Costa JS, Pattussi MP. Fatores associados com a incapacidade funcional em idosos do município de Guatambu, Santa Catarina, Brasil. Cad. de Saúde Pública. 2007;23(11):2781-8. http://dx.doi.org/10.1590/S0102$311 \times 2007001100025$

19. Silva DNN. A influência do meio ambiente no histórico de quedas em idosos brasileiros: revisão integrativa [trabaIho de conclusão de curso] [Internet]. Brasília: Universidade de Brasília; 2017. Disponível em: https://bdm.unb.br/bitstream/10483/20629/1/2017_DanielleBrasilBarrosdaSilva.pdf

20. Conte EMT, Lopes AS. Qualidade de vida e atividade física em mulheres idosas. RBCEH [Internet]. 2005:2(1):61-75. Disponível em: http://seer.upf.br/index.php/rbceh/article/view/23

21. Freitas FAS, Santos ESS, Pereira LSM, Lustosa LP. Vulnerabilidade física de idosos na alta hospitalar. Fisioter. Pesqui. 2017;24(3):253-8. https://doi.org/10.1590/18092950/16205224032017

22. Ciosak SI, Braz E, Costa MFBNA, Nakano NGR, Rodrigues J, Alencar RA, et al. Senescência e senilidade: novo paradigma na atenção básica de saúde. Rev. esc. enferm. USP. 2011;45(2):17638. https://doi.org/10.1590/S0080-62342011000800022 